Rolando Pablo Juárez, 1 Rodolfo Martín Barrere. ${ }^{2}$

${ }^{1}$ Profesor Titular de la Asignatura Fisiología Humana. Coordinador del Módulo Morfofunción II. Facultad de Odontología. Universidad Nacional del Nordeste.

${ }^{2}$ Director del Centro de Estudios sobre Ciencia, Desarrollo y Educación Superior - REDES (Unidad asociada al CONICET) y Secretario Técnico de la Red de Indicadores de Ciencia y Tecnología (RICYT).

Trabajo recibido: 05 de Junio de 2017. Aprobado: 08 de Agosto de 2017.

\section{ESTUDIO BIBLIOMÉTRICO DE LA PRODUCCIÓN CIENTÍFICA ARGENTINA EN CÁNCER A TRAVÉS DE LAS BASES DE DATOS MEDLINE Y LILACS}

BIBLIOMETRIC STUDY THROUGH MEDLINE AND LILACS DATABASES OF SCIENTIFIC ARGENTINIAN PRODUCTION ON CANCER

ESTUDO BIBLIOMÉTRICO DA PRODUÇÃO CIENTÍFICA ARGENTINA EM CÂNCER ATRAVÉS DOS BANCOS DE DADOS MEDLINE E LILACS

\section{Resumen}

Este artículo analiza el perfil de la producción científica argentina en el campo del cáncer entre 2001 y 2010. Una investigación bibliométrica se realizó a través de las bases de datos Medline y Lilacs.

En Medline, se registraron 1132 documentos. El núcleo de Bradford estuvo conformado por 37 revistas con 379 publicaciones. El Instituto de Oncología Ángel H. Roffo (UBA) fue la institución más productiva (71 documentos). En Lilacs, se registraron 824 documentos. El núcleo de Bradford estuvo conformado por cinco revistas con 291 publicaciones. El Hospital Italiano (CABA) fue la institución más productiva (86 documentos).

La investigación en cáncer en Argentina se está incrementando, existe una fuerte producción en las Universidades Nacionales (Medline), Hospitales nacionales y provinciales (Lilacs), con importante participación en investigaciones observacionales descriptivas.

Palabras Clave: bibliometría, cáncer, publicación, Argentina. 


\section{Abstract:}

This article analyzes the profile of Argentinian scientific production in the field of cancer between 2001 and 2010. Bibliometric research was carried out through Medline and Lilacs databases.

In Medline, 1132 documents were registered. Bradford nucleus was made up of 37 journals with 379 publications. The most productive institution (71 documents) was Instituto de Oncología Ángel H. Roffo (UBA). In Lilacs, 824 documents were registered. Bradford nucleus was made up of 5 journals with 291 publications and the most productive institution (86 documents) was Hospital Italiano (CABA).

Cancer research is increasing in Argentina; there is a large production in National Universities (Medline) and in national and provincial Hospitals (Lilacs), with important participation in descriptive observational research.

Key Words: Bibliometrics, Cancer, Publication, Argentina.

\section{Resumo:}

Este artigo analisa o perfil da produção científica argentina no campo do câncer entre 2001 e 2010. Uma pesquisa bibliométrica foi realizada através dos bancos de dados Medline e Lilacs.

No Medline, 1132 documentos foram registrados. O núcleo de Bradford consistiu em 37 revistas com 379 publicações. O Instituto de Oncologia Angel H. Roffo (UBA) foi a instituição mais produtiva (71 documentos). Em Lilacs, foram registrados 824 documentos. $\mathrm{O}$ núcleo de Bradford consistiu em cinco revistas com 291 publicações. O Hospital Italiano (CABA) foi a instituição mais produtiva ( 86 documentos).

A pesquisa em câncer na Argentina está aumentando, há uma forte produção nas Universidades Nacionais (Medline), Hospitais Nacionais e Provinciais (Lilacs), com importante participação em investigações observacionais descritivas .

Palavras chave: bibliometria, câncer, publicação, Argentina.

\section{Introducción}

En las denominadas ciencias de la vida y de la salud, la cuantificación y posterior valoración de la producción científica escrita ocupa un lugar preeminente. En la salud confluyen lo biológico y lo social, el individuo y la comunidad, la política social y la económica. Como toda tradición viva, la salud debe estar en continuo proceso de construcción y renovación. (1)

El análisis bibliométrico no sólo permite examinar retrospectivamente cómo se han logrado y dado a conocer los avances científicos y evaluar el potencial de investigación de las instituciones involucradas, sino que además permite caracterizar el desarrollo de disciplinas científicas y sus líneas de investigación, y las publicaciones científicas en un área del conocimiento, su obsolescencia y dispersión.(2)

Esta información, es una herramienta útil para el análisis de la inversión en ciencia y tecnología de la salud, que complementada con otros indicadores socioeconómicos y la opinión de expertos, permitirán la toma de decisiones para la elaboración de las correspondientes políticas públicas sobre investigación y desarrollo, que impulsen el proceso innovador y fomenten la investigación en el sector sanitario.(3)

Se ha demostrado que la aplicación de métodos bibliométricos, ha sido satisfactoria para el desarrollo de iniciativas de salud públicas para enfermedades específicas, como la diabetes y tuberculosis. $(4,5)$

El cáncer es un problema de salud pública mundial. Para el 2008 se estimó 12,7 millones de 
casos de cáncer y 7,6 millones de muertes por esta causa, de los cuales el 64\% ocurrieron en países en desarrollo.(6)

En Argentina, en el periodo 2001-2010 los tumores registraron una tasa bruta de mortalidad (TBM)promedio de 151 por 100.000 habitantes, la segunda causa de las defunciones que se produjeron en el país, luego de las enfermedades cardiovasculares. En el mismo periodo, el promedio de los años potenciales de vida perdidos (APVP) cada 10.000 habitantesfue de 107, la segunda causa de la muerte de personas jóvenes o de fallecimientos prematuros, luego de causas externas.(7)

El cáncer no es una sola enfermedad, sino cerca de 200 posibles variaciones de una condición progresiva, multifactorial, muy evolutiva, ligada a muchas variables individuales, ambientales y culturales. Es un problema de alta complejidad, y requiere de intervenciones de alta complejidad.(8)

Aunque, la bibliometría es una herramienta útil para evaluar la producción científica, pocos son los estudios bibliométricos que han sido conducidos en base a los resultados de las investigaciones sobre cáncer, en los países de habla hispana de América. La mayoría evalúan distintos puntos sobre la oncología según países, por ejemplo vinculados a Puerto Rico, Perú, México o Colombia, entre otros.(9-12)

La investigación requiere de políticas que ayuden a definir las líneas de investigación y que se favorezca la producción de conocimiento en las áreas que sean de interés para los estados.(13)

Los objetivos principales de este estudio bibliométrico fueron describir la producción científica escrita sobre cáncer en Argentina y generar una línea de investigación documental que sirva como insumo de base para la gestión de políticas de C\&T y políticas de salud referidas al cáncer.

\section{Materiales y Métodos}

Se realizó un estudio observacional, descriptivo, retrospectivo, con descripción cuantitativa y cualitativa de los datos, mediante el análisis de documentos.

La construcción del mapa bibliométrico de la investigación realizada sobre cáncer en Argentina, se realizó entre noviembre de 2010 y marzo de 2013.

Las fuentes de información fueron las bases de datos Medline y Lilacs. Las unidades de análisis fueron las comunicaciones científicas escritas firmadas por autores de instituciones argentinas, sobre la temática cáncer, publicados durante el periodo 2001-2010.

La búsqueda de publicaciones científicas de autores argentinos en ambas bases de datos, se realizó durante el periodo noviembre 2010-noviembre 2011. En Medline el filtro metodológico estuvo constituido por combinaciones de descriptores: "Neoplasia [MAJR] AND Argentina [AD] OR Argentine [AD] AND 2001:2010 [DP]". En Lilacs la selección del campo "descriptor de asunto", nos permitió realizar una búsqueda eficiente para los términos "cáncer" y "neoplasia benigna". En otros campos, "país de afiliación", "año de publicación", utilizando su índice, seleccionamos Argentina durante el periodo 2001-2010. Revisando el resumen de cada comunicación científica, se seleccionaron para el análisis aquellos que se referían a neoplasias malignas.

Los límites de esta investigación, están dados por la cobertura documental de ambas bases de datos, que no registran todas las publicaciones científicas argentinas en cáncer, siendo la cantidad de revistas indizadas, los factores que relativizan este estudio.

Nuestras dimensiones/variables de estudio fueron la extensión bibliométrica (total de artículos), el crecimiento de la documentación (tasa de crecimiento), el índice de actividad (productividad por cantidad de investigadores, productividad en relación a la inversión en I+D),indicador de producción (productividad por número de habitantes, en relación a la población económicamente activa, por PBI, de los autores, por institución, por sector económico y por estado provincial), indicadores de colaboración científica, 
revistas científicas, tipos de publicaciones científicas, tipos de investigaciones, idiomas de publicaciones, localización del cáncer en el cuerpo.

Para el análisis de los datos, se utilizaron métodos estadísticos descriptivos, frecuencias absolutas y relativas. Para conocer la evolución de la extensión bibliométrica se segmentó el período de estudio en dos lustros: 1)2001- 2005 y 2) 2006-2010. Las variables se describieron mediante media y desviación estándar, con intervalos de confianza de $95 \%$ (IC95\%). Para comprobar la significación de la diferencia de medias se utilizó la prueba de la $t$ de Student. El estudio de la relación entre ambas bases de datos se realizó mediante el análisis de correlación bifactorial de Pearson. El nivel de significación utilizado en los contrastes de hipótesis fue de $\mathrm{P} \leq 0,05$. Para el análisis de los datos se utilizó el programa SPSS versión 19 para MS Windows.

\section{Resultados}

En la Tabla N ${ }^{\circ} 1$, se observan las publicaciones en ambas bases de datos, en la década 20012010, sobre ciencias de la salud, total neoplasias, neoplasias benignas, cáncer. En Medline, las publicaciones sobre cáncer representan el 5.02\% del total, en Lilacs el 6.35\% del total.

Tabla Nº1: Publicaciones de las ciencias de la salud, neoplasias y cáncer Bases de Datos - Periodo 2001-2010.

\begin{tabular}{l|c|c}
\hline \multicolumn{3}{c}{ Bases de Datos - Periodo 2001-2010 } \\
\hline Total publicaciones & Medline & Lilacs \\
\hline Total Neoplasias & 22542 & 12982 \\
\hline Benignas & 1508 & 966 \\
\hline Cáncer & 376 & 142 \\
\hline
\end{tabular}

La evolución del número de publicaciones científicas sobre cáncer en la década, no fue constante en ninguna de las bases de datos, (Figura $\mathrm{N}^{\mathrm{o}} 1$ ). Mientras Medline, muestra un crecimiento desparejo, en Lilacs sufrió una disminución en el número de publicaciones.

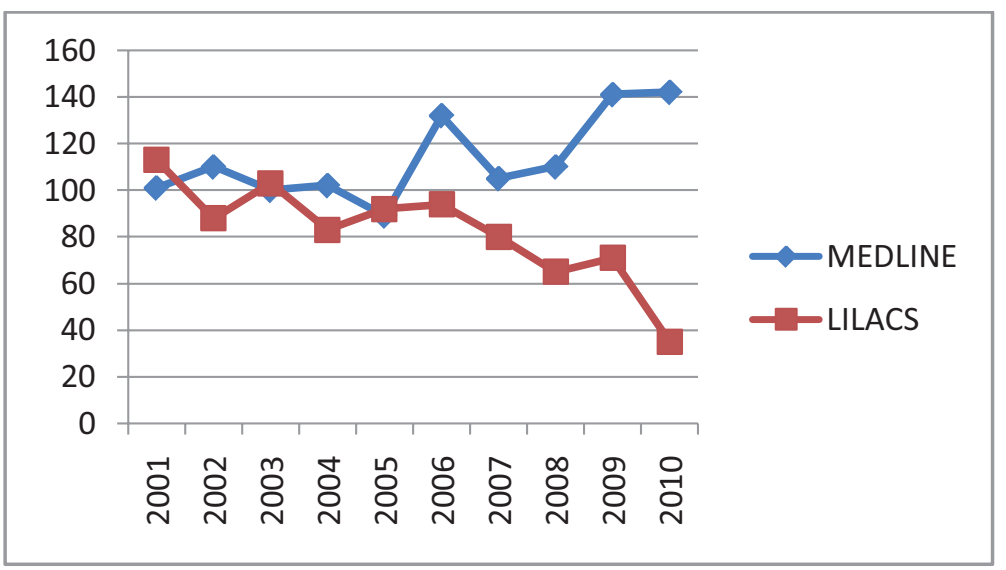

Figura $\mathrm{N}^{\circ} 1$. Evolución del número de publicaciones científicas sobre cáncer

En Medline, las medias y desviación estándar del lustro $1(100,40 \pm 7,5)$ y del lustro 2 $(126,00 \pm 17,4)$ no mostraron diferencias significativas $(\mathrm{p}=0,069)$. 
En Lilacs, las medias y desviación estándar del lustro $1(95,80 \pm 12,1)$ y del lustro $2(69,00$ $\pm 21,9)$ mostraron diferencias significativas $(\mathrm{p}=0,043)$.

Al comprobar, la significación de la diferencia de medias de toda la década, Medline $(113,20 \pm 18,4)$ - Lilacs $(82,40 \pm 21,8)$, se obtuvieron diferencias significativas $(\mathrm{p}=0,026)$. La correlación fue negativa $(\mathrm{r}=-0,663 ; \mathrm{p}=0,051)$.

En la Tabla $\mathrm{N}^{\circ} 2$, se puede apreciar el índice de actividad y de producción por número de habitantes, en relación a la población económicamente activa y por PBI.

Tabla N²: Promedio del índice de actividad (IA) e indicador de producción (IP).

\begin{tabular}{l|c|c}
\hline \multicolumn{3}{c}{ Bases de Datos - Periodo 2001-2010 } \\
\hline & Medline & Lilacs \\
\hline IA por cada 100 investigadores & 2.0 & 1.5 \\
\hline $\begin{array}{l}\text { IA por cada millón de pesos de } \\
\text { inversión en I + D* }\end{array}$ & 0.3 & 0.3 \\
\hline IP por 100000 habitantes & 0.3 & 0.2 \\
\hline IP por cada millón de PEA** & 7.1 & 5.2 \\
\hline IP por cada millón de PBI*** & 0.2 & 0.2 \\
\hline
\end{tabular}

*Investigación y Desarrollo, **Población Económicamente Activa, ***Producto Bruto Interno

El número total de autores principales, principales asociados o coautores, en Medline fue de 6674, en Lilacs de 3757.

Los autores argentinos personales con mayor producción, en el periodo 2001-2010, figurando como autores principales, principales asociados o como coautores tuvieron un promedio de 14 publicaciones en Medline, con un máximo de 25 en un autor. En Lilacs, el promedio fue de 9 publicaciones, con un máximo de 16 en un autor.

Con respecto a la productividad de los autores, sobre la base del índice de Lotka, se distribuyó a los autores en tres niveles de productividad (Tabla $\mathrm{N}^{\circ} 3$ ). En la base de datos Medline, predominaron los trabajos que poseían entre cuatro y ocho autores, en Lilacs los que tenían autoría simple y entre cuatro y seis autores.

Tabla N³: Indicador de producción por autores.

\begin{tabular}{l|c|c}
\hline \multicolumn{3}{c}{ Bases de Datos - Periodo 2001-2010 } \\
\hline & Medline & Lilacs \\
\hline Pequeños productores* & $82.39 \%$ & $85.53 \%$ \\
\hline Medianos productores** & $16.54 \%$ & $14.37 \%$ \\
\hline Grandes productores*** & $1.07 \%$ & $0.10 \%$ \\
\hline
\end{tabular}

*Con un único trabajo e índice de productividad igual a 0 , **entre 2 y 9 trabajos, e índice de productividad mayor que 0 e igual a $1, * * *$ entre 10 o más trabajos e índice de productividad igual o mayor que 1.

En productividad por institución, en Medline la mayor producción se observó en Institutos, Facultades y Hospitales dependientes de la Universidad Nacional de Buenos Aires. El Instituto de Oncología Ángel H. Roffo (UBA) fue la institución más productiva (71 documentos). En Lilacs, la mayor producción se apreció en Institutos y Hospitales privados. El Hospital Italiano (CABA) fue la institución más productiva ( 86 documentos).

En productividad por sector económico, en Medline existió una fuerte producción en las Universidades Nacionales, Hospitales nacionales y provinciales, los Institutos de C\&T;las empresas privadas y las instituciones sin fines de lucro mostraron una escasa producción. En Lilacs, existió una fuerte producción en los Hospitales nacionales y provinciales, en 
empresas privadas. Las instituciones sin fines de lucro mostraron una escasa producción. En productividad por estado provincial, en ambas bases de datos, la Ciudad Autónoma de Buenos Aires, presentó el mayor número de publicaciones. Buenos Aires, Córdoba y Santa Fe, exhibieron un número moderado. El resto de las unidades federales, presentaron muy pocas publicaciones.

Con respecto a los indicadores de colaboración científica, en Medline, la cooperación nacional predominó entre institutos científicos y unidades académicas con un 47.66\%; la cooperación internacional, se formalizó a través de hospitales públicos en un 59.09\%. En Lilacs, la cooperación nacional prevaleció entre hospitales públicos y unidades académicas con un $19.17 \%$, hospitales públicos y empresas $16.43 \%$; la cooperación internacional, se plasmó a través de centros privados en un $40.74 \%$.

En Medline, las revistas más prolíficas en la zona llamada núcleo de la distribución de Bradford, reúnen el $33.48 \%$ de las publicaciones sobre cáncer: 379 publicaciones en 37 revistas, 24 especializadas en cáncer. La revista con mayor número de publicaciones fue Medicina (Buenos Aires)con 71 publicaciones.

En Lilacs, el 35.31\% de los trabajos sobre cáncer, con un total de 291 publicaciones, están concentrados en cinco revistas científicas, una especializada en cáncer. La revista con mayor número de publicaciones fue Revista Argentina de Cirugía con 85 publicaciones.

En categorías de documentos, en ambas bases de datos, existió un predominio de documentos con formato IMRYD (artículo científico), en Medline con un $71.73 \%$ y Lilacs con un $41.14 \%$. En Medline, los formatos revisión alcanzaron un 9.4\% y reporte de casos clínicos $16.87 \%$; en Lilacs, alcanzaron un $18.45 \%$ y $27.91 \%$, respectivamente.El resto de los documentos, en Medline $1.9 \%$ y en Lilacs $12.5 \%$.

La distribución de las investigaciones, según su diseño experimental, se aprecia en la Tabla 4. En Medline se registraron 24 estudios multicéntricos, en Lilacs se registró un estudio multicéntrico.

Tabla No4: Diseño experimental investigaciones.

\begin{tabular}{l|c|c}
\hline \multicolumn{3}{c}{ Bases de Datos - Periodo 2001-2010 } \\
\hline Observacionales descriptivas & Medline & Lilacs \\
\hline Ensayos clínicos & $79.31 \%$ & $91,17 \%$ \\
\hline Estudios analíticos* & $9.6 \%$ & $1,4 \%$ \\
\hline \multicolumn{2}{c}{${ }^{*}$ Cohorte, casos y controles }
\end{tabular}

La distribución de las investigaciones en Medline, según la clasificación de Frenk et al. (1986),(14)fue: las de mayor número las investigaciones clínicas con un $59.77 \%$, seguidas por las biomédicas $32.73 \%$ y las de salud pública $7.5 \%$.En Lilacs, investigaciones clínicas $59.88 \%$, biomédicas $23.30 \%$ y las de salud pública $16.82 \%$.

En Medline, predominaron las publicaciones en inglés con un $86.75 \%$, seguidas por el español $12.63 \%$ y español-inglés con $0.62 \%$. En Lilacs, prevalecieron las publicaciones en español con un $96.60 \%$, seguidas por el inglés $3.28 \%$ y portugués con $0.12 \%$.

En Medline, los cánceres más estudiados fueron cáncer de mama, hematológico, piel, colon y recto, pulmón, orofaríngeo, sistema nervioso, útero. En Lilacs, fueron cáncer de mama, colon y recto, piel, orofaríngeo, pulmón, útero.

En la Figura $\mathrm{N}^{\circ}$ 2, se observa la distribución de las publicaciones en Medline y Lilacs, según localización del cáncer en el cuerpo humano y/o animal y su relación con la TBM. 


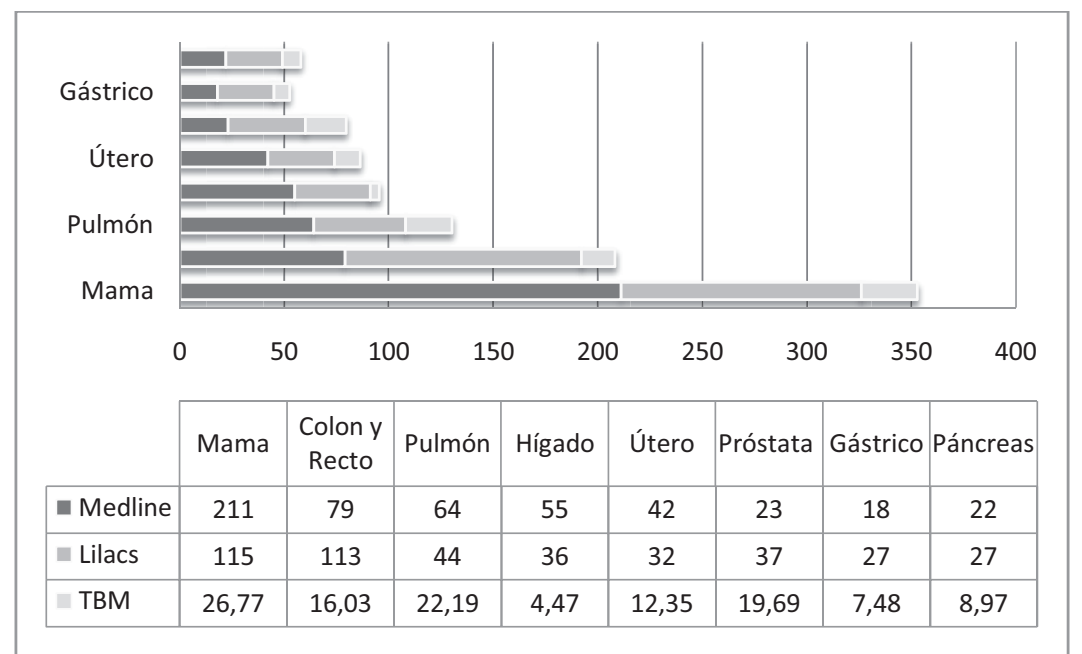

TBM. Tasa bruta de mortalidad (por 100.000 habitantes), según grupos de causas seleccionadas.

Figura $\mathrm{N}^{\circ}$ 2. Número de publicaciones científicas sobre cáncer y TBM según localización del cáncer en el cuerpo

\section{Discusión}

Este es el primer estudio realizado en Argentina, que combina datos bibliométricos y epidemiológicos para analizar la problemática del cáncer. Este enfoque, permite una evaluación de la relación, entre producción biomédica de la investigación del cáncer y tasa de mortalidad del cáncer, en la población argentina.

Las posibilidades de aplicación de los indicadores bibliométricos están en relación directa con la informatización de los documentos y las ventajas ofrecidas por las bases de datos. (15) En nuestro estudio, la gran capacidad de almacenamiento de Medline, permitió actuar sobre grandes unidades de datos, en cantidades suficientes para una evaluación correcta. Entre 2001 y 2010, un total de 277.957 artículos, se publicaron en todo el mundo, en 163 revistas especializadas en oncología e indizadas en Medline. El número de los artículos de los EE.UU., contribuyó con el 31.4\% (87201/277957) del total, seguido por Japón 8.6\%, Alemania 5.7\%, Reino Unido 4.8\%, China $4.5 \%$ y Francia 3.7\%.(16)Teniendo en cuenta estos datos, y las 1132 publicaciones sobre cáncer que obtuvimos en Medline, hemos calculado que Argentina contribuye con un $0.4 \%$.

Nuestros hallazgos, indican un creciente interés en la investigación del cáncer en Argentina. El aumento del número de artículos científicos relacionados con cáncer en la década estudiada, en la base de datos Medline, está en consonancia con otros estudios a nivel América Latina, que han demostrado un aumento en la publicación de la investigación del cáncer en diversos países de la región. $(9,17,18)$

El rápido aumento de la prevalencia mundial del cáncer y su impacto económico en los sistemas de salud, tanto en países desarrollados como en los países en vía de desarrollo, pueden justificar el incremento en las referencias bibliográficas, debido a la necesidad de aportar conocimiento sobre la problemática planteada. (19)

La disminución del número de publicaciones en Lilacs, a partir del año 2007, se debe a que la metodología Lilacs, provocó un retraso en la incorporación de nuevas publicaciones o números anteriores. Una nueva búsqueda realizada en diciembre de 2012 en Lilacs, arrojó un aumento de publicaciones, en toda la década, pero principalmente en el periodo 20072010. Entre la primera y la segunda búsqueda bibliográfica, la diferencia en el periodo 2001-2006 fue de 68 publicaciones, mientras que en el periodo 2007-2010 fue de 133. En 
el año 2010, la diferencia fue de 50 publicaciones.

En la década estudiada, el número de publicaciones científicas sobre cáncer en relación al número de habitantes, se mantuvo estable en ambas bases de datos; diferenciándose de otros países que, en el periodo 2000-2008 aumentaron, como USA, Japón, Brasil y Unión Europea, siendo China el más destacado. (20)

Entre los años 2001 y 2010, Argentina ha disminuido el número de publicaciones sobre cáncer en Medline y Lilacs, en relación al PIB. Otros países, que han disminuido su producción en el periodo 2000-2008 y en relación al PBI, son: los de la Unión Europea y Rusia. Aumentaron, en forma despareja: Japón, Brasil y Méjico. Aumentaron sostenidamente: USA y China.(20)

Como un parámetro adicional asociado con el crecimiento de las publicaciones en Medline, la cantidad de investigadores también creció a partir de 2004-2005, (información elaborada a partir de los datos del MINCYT, Indicadores de C\&T 2001-2010).

Sin embargo, no presentaron un nivel de producción que los posicionara internacionalmente. Por cada cien investigadores pertenecientes a las ciencias médicas, en el período 20012010, las publicaciones sobre cáncer no superan los 2.0 documentos en revistas indizadas en Medline, respectivamente; mientras que, por cada 100 investigadores del sistema científico argentino, las publicaciones sobre salud los promedios fueron de 5.3.

La comunidad científica de la medicina argentina, no asumió en la década estudiada, la investigación en cáncer como el objeto central de su producción científica, representando solo el $5 \%$ en Medline y $6.3 \%$ en Lilacs, superada por Chile donde en Medline alcanza el 7,3\% y en Lilacs el 9.8\% (búsqueda propia).

En la base de datos Medline, predominaron los trabajos que poseen entre cuatro y ocho autores, con un $65 \%$ del total. En Lilacs, los que tuvieron entre cuatro y seis autores, con un $39 \%$ del total. Datos coincidentes con revistas científicas internacionales, dondelos artículos biomédicos tienen un índice de coautoría cercano a 5.(21)

Esas cifras, indicarían un desarrollo de la bibliometría argentina sobre cáncer, en dirección a la especialización. Cuando se considera el ciclo de desarrollo de cualquier disciplina, al inicio la colaboración entre los autores (la co-autoría) es nula o baja, pero la proporción de autores únicos es alta. Conforme la disciplina se desarrolla comienza la profesionalización, la disciplina se abre a mayores exploraciones y se inicia la especialización. Entonces, los autores comienzan a colaborar y la proporción de autores únicos comienza a decrecer, y al contrario, las publicaciones en colaboración comienzan a crecer.(22)

Siguiendo los parámetros de la Ley de Lotka, en ambas bases de datos predominaron los autores designados como "pequeños productores", con un único trabajo e índice de productividad igual a 0. En Medline, encontramos el 82.39\% con único trabajo, en Lilacs $85.53 \%$. Una aplicación práctica del cumplimiento o no de esta ley, podría traducirse en la asignación de fondos gubernamentales para el desarrollo de un área científica, como podría ser la temática del cáncer. En todo caso, la política gubernamental debiese ser la asignación de fondos equivalentes, tanto a los grandes como a los pequeños productores.

Las publicaciones sobre cáncer en Medline surgieron del sector gubernamental en un $85.44 \%$, organismos públicos $44.23 \%$ y universidades públicas $41.21 \%$, y del sector privado en un $14.56 \%$, entidades sin fines de lucro $9.22 \%$ y empresas $5.34 \%$. Las publicaciones sobre cáncer, en Lilacs y en el mismo periodo surgieron del sector gubernamental en un $61.46 \%$, organismos públicos $38.13 \%$ y universidades públicas $23.33 \%$ y del sector privado en un $38.54 \%$, entidades sin fines de lucro $9.79 \%$ y empresas $28.75 \%$.

Estos datos, coinciden con las aportaciones españolas sobre salud en la literatura internacional, mostrando que la mayoría de los trabajos proceden de la Universidad, pero también las instituciones de la sanidad pública, experimentan un intenso crecimiento como centros en los que trabajan los autores de los trabajos.(24)

Los vínculos universidad-industria, en los países desarrollados no fueron creados al azar, o 
por las fuerzas del mercado solamente; en muchos de ellos, hubo una acción deliberada del Estado como asunto de política de Estado, con el fin de promover una relación mutuamente benéfica entre los centros de investigación (usualmente las universidades) y las empresas privadas (y públicas). (25)

En nuestro trabajo, las empresas se refieren a clínicas, sanatorios y hospitales privados, pero también a empresas farmacéuticas y biotécnicas. Estas últimas, muestran una escasa producción: 12 en Medline y una en Lilacs. Esto amplía, la necesidad de búsqueda de mecanismos apropiados que incentiven al sector privado, para incrementar sus inversiones en $\mathrm{I}+\mathrm{D}$ en el área oncológica, con la finalidad de reducir la brecha con los países desarrollados.

La distribución de las publicaciones científicas sobre cáncer, por unidad federal, mostró en Medline que la Ciudad Autónoma de Buenos Aires es la primera productora con un $65.54 \%$, seguida por la Provincia de Buenos Aires 17.13\%, Córdoba 8.12\%, Santa Fe $3.6 \%$, el resto de las provincias 5.61\%. En Lilacs, la Ciudad Autónoma de Buenos Aires es la primera productora con un 70.14\%, seguida por la Provincia de Buenos Aires 10.80\%, Córdoba $8.13 \%$, Santa Fe $1.94 \%$, el resto de las provincias $8.28 \%$.

En el área nacional, se da una concentración de autores e instituciones en la Ciudad Autónoma de Buenos Aires, quizá excesiva en un primer análisis, pero lógica por la cantidad de centros existentes en esta ciudad frente a los que, proporcio $\neg$ nalmente, se cuentan en otras zonas. Cuanto más nos alejamos del centro geográfico, más disminuyen las cifras y, por tanto, más esporádicas son las colabora $\neg$ ciones de otras comunidades.

La colaboración científica, está aumentando en la mayoría de las disciplinas, debido entre otros factores, a la creciente complejidad de los proyectos de investigación, para los cuales se requieren cada vez más un gran número de investigadores especializados en las diferentes áreas. Una mayor colaboración, puede conducir a una mayor calidad de los resultados debido a la experiencia colectiva y el trabajo en conjunto de los científicos.(26) La UBA, fue la institución con mayor número de colaboraciones en LILACS; sin embargo, estas son de poca intensidad, publica colaborativamente con muchas instituciones, pero estas colaboraciones generan pocos artículos en común. Esto podría deberse a varios factores, como el que la intensidad de las colaboraciones depende del tiempo de trabajo en conjunto y el desarrollo de investigaciones clínicas en cáncer requiere largos tiempos de seguimiento.

En Medline, la UBA también mostró mayor número de colaboraciones, pero a diferencia de Lilacs estas fueron intensas, específicamente en asociación con el CONICET. Esto podría, deberse a una fuerte política de articulación entre ambas instituciones, establecida desde el CONICET, a través de Institutos compartidos y Unidades ejecutoras que consolidaron los grupos de investigación.

El comportamiento de la investigación en cáncer a nivel mundial, muestra una mayor producción en cáncer de mama, pero muy pobre en otros tipos de cáncer que generan alta mortalidad, generando una pobre asociación entre la carga de enfermedad por cáncer y las investigaciones realizadas. $(10,27,28)$ Nuestros resultados, respaldan en parte el argumento de que, en relación con su impacto en la sociedad(TBM), hay cánceres subrepresentados. En nuestro trabajo, el mayor desarrollo de las investigaciones en las áreas clínicas y biomédicas, refleja la tendencia mundial para el desarrollo e impulso de la medicina personalizada, enfocada en líneas de diagnóstico, tratamiento y ciencias básicas. $(12,17,29)$

\section{Conclusiones}

Los resultados obtenidos, muestran que Argentina tiene una actividad científica en producción sobre cáncer moderada, sí la comparamos con el total de las Ciencias de la Salud y con otros países de la región (Brasil) y del mundo (EEUU y China).

Se ha observado un desequilibrio regional en la producción científica de esta disciplina, 
puesto que sólo una unidad federal produce casi el $65-71 \%$ de los documentos.El sector público, fue uno de los principales agentes de producción.

Los estudios observacionales descriptivos, representan un importante porcentaje de las investigaciones en cáncer. Se investiga más, en cáncer de mama, colorrectal y pulmón, guardando relación directa con la TBM en el cáncer mamario e inversa en las localizaciones pulmón, colon y recto.

La creación del MINCYT y del Instituto Nacional del Cáncer (INC), en los últimos años del presente estudio, mostró una reactivación de las políticas científicas y sanitarias del cáncer, mostrando un interés en integrar los diferentes actores sociales involucrados en la temática.

El control de las enfermedades malignas, requiere datos científicos (investigación oncológica-bibliometría) y una gestión competente (políticas públicas).Desde la perspectiva CTS, el establecimiento de programas nacionales del control del cáncer, requiere la articulación de políticas de ciencia y tecnología con las de salud.

Además, de orientar las investigaciones clínicas y biomédicas, a través de la relación carga de la enfermedad (TBM, APVP) y la especificidad de sitio (localización del cáncer en el cuerpo),será necesario fomentar líneas de investigación con pertinencia social relacionadas con opciones programáticas que permitan reducir el riesgo de cáncer y mejorar la detección temprana.

El INC, es una oportunidad para crear y fortalecer redes de investigación en cáncer a nivel regional, nacional e internacional, y los convenios que se vienen realizando para trabajo en conjunto, verán reflejados sus efectos en futuras publicaciones.

\section{Bibliografía}

1. Rousseau R. Journal Evaluation: Technical and Practical Issues. Libr Trends. 2002;50(3):418-439.Disponible en: http://www.zabgu.ru/files/Rousseau2002.pdf

2. Sanz-Valero J, Tomás Casterá V, Wanden-Berghe C. Estudio bibliométrico de la producción científica publicada por la Revista Panamericana de Salud Pública 1997 a 2012. Rev Panam Salud Publ. 2014;35(2):81-8. Disponible en: http://iris.paho. org/xmlui/bitstream /handle/123456789/8465/a01v35n2.pdf?sequence $=1$

3. Alvis-Guzmán N, De La Hoz-Restrepo F. Producción Científica en Ciencias de la Salud en Colombia, 1993-2003. Rev de Salud Pública (Bogotá, Colombia). 2006;8(1):25-37.Disponible en: http://www.scielosp.org/scielo.php?script=sci_artt ext\&pid=S0124-00642006000100003

4. Arunachalan S, Gunasekaran S. Tuberculosis research in India and China: from bibliometrics to research policy. Curr Sci. 2002; 82(8):933-47. Disponible en: http://www.iisc.ernet.in /currsci/apr252002/933.pdf

5. Arunachalan S, Gunasekaran S. Diabetes research in India and China today: from literature-based mapping to healthcare policy. CurrSci. 2002;82(9):1086-97. Disponible en: http:/arizona.openrepository.com/arizona/handle/10150/105930

6. Jemal A, Bray F, Center MM, Ferlay J, Ward E, Forman D. Global cancer statistics. CA Cancer J Clin. 2011;61(2):69-90.doi:10.3322/caac.20107.

7. Ministerio de Salud. Presidencia de la Nación. Indicadores Básicos Argentina. Ministerio de Salud de la Nación (Dirección de Estadísticas e Información en Salud) y la Organización Panamericana de la Salud (Datos y Estadísticas). Disponible en: http://www.msal.gov.ar y http://www.paho.org/arg

8. Jiménez, J. (2010). Mapa de la Investigación del Cáncer en Chile. Consorcio Tecnológico en Biomedicina Clínico-Molecular Aplicada (BMRC-Chile), Programa de Salud Pública y Centro de Evaluaciones Económicas y Sociales en Salud Departamento de Salud Pública, Pontificia Universidad Católica de Chile. Disponible en: http://www.saludunderecho.cl /archivos/2010/12/Mapa-del- 
C\%C3\%A1nceren-Chile-2010.pdf

9. Ortiz AP, Calo WA, Suárez-Balseiro C, Maura-Sardo M, Suárez E. Bibliometric assessment of cancer research in Puerto Rico, 1903-2005. Rev Panam Salud Públ. 2009;25(4):353-61.Disponible en: https://www.ncbi.nlm.nih.gov/pmc/articles/ PMC3031111/

10. Mayta-Tristán P, Huamaní C, Montenegro-Idrogo JJ, Samanez-Figari C, GonzálezAlcaide G. Producción científica y redes de colaboración en cáncer en el Perú 20002011: un estudio bibliométrico en Scopus y ScienceCitationIndex. Rev PeruMedExp Salud Publica. 2013;30(1):31-6. Disponible en: http://www.scielo.org.pe/scielo. php?pid=S1726-46342013000100006 \&script =sci_arttext

11. Pérez-Santos JL, Anaya Ruiz M. Mexican breast cancer research output, 20032012. AsianPac J CancerPrev. 2013;14(10):5921-3. Disponible en: http://journal. waocp.org/article_28230_523af5760e0bf44 cb5de184ca64d3c41.pdf

12. Carreño Dueñas A, Rojas MP, Lucio-Arias D, ML Serrano, Piñeros M. Investigación en cáncer en Colombia, 2000-2010. Rev ColombCancerol. 2015;19(1):39-46. Disponible en: http://www.scielo.org.co/ pdf/rcc/v19n1/v19n1a06.pdf

13. Lewison G, Purushotham A, Mason M, McVie G, Sullivan R. Understanding the impact of public policy on cancer research: a bibliometric approach. Eur J Cancer. 2010;46(5):912-9.doi: 10.1016/j.ejca.2009.12.020.

14. Frenk J, Bobadilla JL, Sepúlveda J, Rosenthal J, Rules E. Un modelo conceptual para la investigación en salud pública. Bol Of SanitPanam. 1986;101(5):477-492.

15. Alzate Piedrahita MV, Arbelaez Gómez MC, Gómez Mendoza MA, Romero Loaiza F. Bibliometría y Discurso Pedagógico. Editorial Papiro: Pereira - Colombia; 2004. Disponible en: http://www.centromanes.org/?page_id=8509\&print=pdf

16. Liu X-Y, Wan X-H, Li Z-W. Ten-year survey on oncology publications from China and other top-ranking countries. Chin Med J. 2011; 124(20):3314-3319.

17. Hofman, K., Ryce, A., Prudhomme, W., \&Kotzin, S. (2006). Reporting of noncommunicable disease research in low- and middle-income countries: a pilot bibliometric analysis. J Med Libr Assoc. 2006;94(4):415-420. Disponible en: https://www.ncbi.nlm.nih.gov/pmc/articles/PMC1629422/

18. Acevedo AM, Gómez A, Becerra HA, Ríos AP, Zambrano PC, Obando EP et al. Distribution and trends of hematology and oncology research in Latin America: A decade of uncertainty. Cancer. 2014;120(8):1237-45.doi: 10.1002/cncr.28539.

19. World Health Organization. The WHO's fight against cancer strategies that prevent, cure and care. Geneva: WHO; 2007. Disponible en: http://www.who.int/cancer/ publicat/WHOCancerBrochure2007.FINALweb.pdf?ua=1

20. Micheli A, Di Salvo F, Lombardo C, Ugolini D, Baili P, Pierotti MA. Cancer research performance in the European Union: a study of published output from 2000 to 2008. Tumori. 2011;97(6):683-689.doi: 10.1700/1018.11081.

21. Camps D. Estudio bibliométrico general de colaboración y consumo de la información en artículos originales de la revista Universitas Médica, período 2002 a 2006. Universitas Médica. 2007;48(4):358-365. Disponible en: http://www.redalyc. org/pdf/2310/231018670002.pdf

22. Urbizagastegui Alvarado R, Restrepo Arango C. Modelando la distribución del número de co-autores por artículo. Investigbibl. 2011; 25 (53): 103-119 [citado 2013-11-04]. Disponible en: <http://www.scielo.org.mx/scielo.php?script=sci arttext\&pid=S0187-358X2011000100005\&lng=es\&nrm=iso $>$

23. Rau Jaime R. (2011). ¿Sigue la producción de artículos ISI de los ecólogos chilenos (sensu lato) la ley de Lotka (1926)?. Rev ChilHistNat [citado 2013 Dic 02]; 84(2): 213-216. Disponible en: http://www.scielo.cl/scielo.php?script=sci_ arttext\&pid=S0716-078X2011000200007

24. Jiménez-Contreras E, Faba C, de Moya Anegón F. El destino de las revistas 
científicas nacionales. El caso español a través de una muestra (1950-90). Rev EspDoc Cient. 2001;24(2):147-61. doi: http://dx.doi.org/10.3989/ redc.2001.v24.i2.47

25. Yusuf, S. University-industry links, policy dimensions. En Y. Shahid y K. Nabeshima (eds.), How universities promote economic growth, Washington, Banco Mundial, Directions in Human Development;2007. Disponible en: http://siteresources.worldbank.org /EDUCATION/ Resources/278200-1121703274255/1439264-1229357921800/Yusuf_ University-Industry_2007.pdf

26. Ortiz-Rivera L, Sanz-Casado E, Suarez-Balseiro CA. Scientific production in Puerto Rico in science and technology during the period 1990 to 1998. Scientometrics. 2000;49(3):403-18.doi: 10.1023/A:1010585522899

27. Saad ED, Pinheiro CM, Masson AL, Borghesi G, Hoff PM, Prisco FE. Increasing output and low publication rate of Brazilian studies presented at the American Society of Clinical Oncology Annual Meetings. Clinics. 2008;63(3):293-6.doi: 10.1590/S1807-59322008000300001

28. Glynn RW, Chin JZ, Kerin MJ, Sweeney KJ. Representation of cancer in the medical literature-a bibliometric analysis. PLoS One.2010;5(11):e13902. doi: 10.1371/journal.pone.0013902.

29. Abernethy A, Abrahams E, Barker A, Buetow K, Burkholder R, Dalton WS, et al. Turning the tide against cancer through sustained medical innovation: the pathway to progress. Clin Cancer Res.2014;20(5):1081-6. doi: 10.1158/1078-0432.CCR-13-3430. 